\title{
LOS MALENTENDIDOS NATURALES EN EL ESTUDIO SOCIOLINGÜÍSTICO DE LA CIUDAD DE MÉXICO*
}

Se produce un malentendido cuando nuestro interlocutor entiende de una manera diferente lo que quisimos decir en realidad. Como se verá, casi siempre que malinterpretamos a alguien oímos algo con cierto sentido, por forzado que sea. Es raro que la interpretación fallida no lo tenga en absoluto. Un malentendido es, entonces, más una bifurcación interpretativa que una vía muerta. Por otra parte, no tiene mucho sentido hablar de malentendidos fuera de conversaciones naturales. Aunque cabe imaginar otras situaciones, el interés de este trabajo se centra en los malos entendidos que aparecen en los encuentros lingüísticos cara a cara ${ }^{1}$.

En (1) y (2) hay un par de ejemplos de malentendidos:

(1) A: ¿Quito la JERGA?

B: No, la TARJETA déjala [58 $]^{2}$.

(2) A: ¿Quién quiere CANDEREL?

B: Aquí, yo pedí CAFÉ [55].

Se trata de materiales muy interesantes para el estudio del contacto entre dialectos y personas, y para la discusión de varios aspectos

* Este trabajo forma parte del proyecto "Estructura fónica de la diversidad lingüística en México" (CONACYT, 27598H). Una versión previa se leyó en el XII Congreso de la Asociación de Lingüística y Filología de la América Latina, Santiago de Chile, agosto de 1999.

${ }^{1}$ Entre 1984 y 1990 Labov y sus colaboradores recogieron casi 697 casos de malos entendidos que habían aparecido de manera natural en la conversación espontánea. "El propósito de la colección es comparar malentendidos de la vida cotidiana con los resultados de los experimentos controlados sobre comprensión trans-dialectal" (Principios del cambio lingüistico, t. 1: Factores internos, trad. P. Martín, Gredos, Madrid, 1996, p. 430).

${ }^{2}$ El número que va entre corchetes al final de cada ejemplo corresponde al asignado a la ficha que le corresponde en la base de datos. Se destaca en versalitas la parte crucial del malentendido. 
cognoscitivos de la variación lingüística ${ }^{3}$. Se ha propuesto que los malentendidos dentro de subsistemas fónicos deberían ser más frecuentes que entre subsistemas, pero esto no es del todo cierto ${ }^{4}$.

Por otra parte, la obtención de este tipo de datos presenta serias dificultades metodológicas, pues no hay manera de grabarlos o preguntarlos. Sólo se puede esperar que ocurran, observarlos y anotar lo que ocurrió de la manera más fiel posible. Aunque todos tenemos experiencias cotidianas de malos entendidos, no es fácil y mucho menos rápido formar una colección representativa de casos. En el marco de nuestro trabajo sociolingüístico sobre la ciudad de México hemos tomado como tarea adicional, entre otras, la de recoger una muestra de malos entendidos en la conversación ${ }^{5}$. La muestra es todavía pequeña, pero crece merced a ciertas dosis de paciencia. Examino a continuación, de entre los materiales ya disponibles, los primeros cincuenta $\operatorname{casos}^{6}$.

\section{MALENTENDIDOS GLOBALES Y MALENTENDIDOS LOCALES}

La estructura normal de los malos entendidos es un nudo, una reparación y una resolución del conflicto. Conviene hacer una primera distin-

${ }^{3}$ En su trabajo aún inédito, "Corpus para logoaudiometría en español: un enfoque lingüístico-frecuencial”, Esther Herrera presenta un experimento de repetición de series de palabras en una muestra de hablantes después de habérselas hecho escuchar. Parece haber varios paralelismos entre los datos obtenidos en una situación experimental como ésta y lo que se documenta en la observación de malos entendidos en conversación espontánea. Confío en que sea posible examinar posteriormente, y con más detalle, las semejanzas y las diferencias entre ambos trabajos.

${ }^{4}$ En los datos de Labov hay 78 casos dentro de subsistemas y 74 entre subsistemas. Un examen más detenido de los datos reveló que la mayor parte de los malentendidos a través de subsistemas se daban en entornos especiales, mientras que ello no ocurría tan abrumadoramente dentro de los subsistemas. Este fenómeno se comprueba también parcialmente para el español. Hay una diferencia esencial con los datos de Labov para el inglés. Allí los malentendidos parecen deberse sobre todo a confusiones con las vocales, aunque a veces involucren también consonantes; los problemas de interpretación del español afectan en primer término a las consonantes.

${ }^{5}$ Véase al respecto Y. Lastra y P. Martín Butragueño, "El modo de vida como factor sociolingüístico en la ciudad de México", en Estructuras en contexto. Estudios de variación lingüística, ed. P. Martín, El Colegio de México, México, 2000, pp. 13-43.

${ }^{6}$ Los malentendidos se registran según los campos previstos en fichas electrónicas con las que se va formando una base de datos. Los principales campos son: transcripción del acontecimiento, descripción de los participantes y de la situación, fecha y una primera evaluación del tipo de malentendido documentado. Uno de los problemas es la lentitud con la que va creciendo la muestra. Podría decirse que hace falta casi un año para reunir un centenar de ejemplos. La parte sustancial del trabajo de Labov se centra en la discusión de 154 casos estrictamente locales (en el sentido definido a continuación). Aprovecho para agradecer la valiosa ayuda de cuantas personas me han dado y me siguen dando ejemplos. 
ción respecto al ámbito en que se produce el malentendido. Fijémonos en los siguientes ejemplos (3 es una conversación telefónica):

(3) P: Que llame a nuestra casa, de parte de Lourdes Y PEDRO.

O: Que llame a su casa, de parte de Lourdes Quintana.

P: No, no, de Lourdes y Pedro, de Lourdes y Pedro [20].

(4) K: Te acuerdas de cómo tiene Marula así sus pelitos, ¿ives cómo tengo yo también? Ya descubrí que es porque se baña para abajo. No me gusta. Mira cómo tengo, no me gusta, siento la frente MENOS LIBRE.

D: ¿ंME LOS LIBRE?

K: Sí, menos libre.

D: ¡Ah! Yo entendí me los libre [2].

En el ejemplo (3) lo que $\mathrm{O}$ ha malentendido no es fruto de una reestructuración fonológica simple. De hecho, parece haber pocos parecidos entre y Pedro y Quintana, más allá del número de sílabas y la asignación del acento. Es difícil establecer las causas de la confusión; (3) es, por tanto, un malentendido global.

Más interesantes por el momento son los casos del tipo (4). Entre menos y me los hay tres diferencias: en primer lugar, $l$ por $n$; en segundo lugar, menos recibe acento primario y me los no; por fin, una diferente asignación de los lindes de palabra. Como es posible determinar mediante reglas relativamente simples la relación entre las contrapartes, se trata de un malentendido local, a pesar de concentrar tres procesos, algunos de ellos bastante habituales. En este trabajo se examinan casi exclusivamente ejemplos de esta segunda clase de confusiones ${ }^{7}$.

La proporción de malentendidos globales es pequeña ${ }^{8}$, unos ocho de entre los primeros cincuenta casos recogidos ${ }^{9}$.

\section{TIPOS DE REGLAS IMPLICADAS EN LOS MALENTENDIDOS}

Se ha propuesto que existen tres tipos principales de reglas sociolingüísticas ${ }^{10}$. Para el caso que interesa, las reglas regulativas sirven para

${ }^{7}$ En realidad, la inmensa mayoría de los ejemplos recogidos hasta ahora son de malentendidos locales. Sin embargo, no es fácil establecer si ello se debe a que, en efecto, sea más raro confundirse globalmente al escuchar lo que alguien nos dijo, o si se trata de un problema de la recolección de los datos: porque los malentendidos globales no se perciban - lo que parece poco probable- o porque el recolector haya concentrado más su atención en los problemas locales - lo cual tampoco es necesariamente cierto.

${ }^{8}$ Será necesario confirmar el sentido de esa proporción.

${ }^{9}$ Son los ejemplos 13, 20, 26, 29, 38, 39, 42, 43.

10 Debe verse el muy sugerente trabajo N. DitTmar, "Descriptive and explanatory power of rules in sociolinguistics", en Towards a critical sociolinguistics, ed. R. Singh, J. Benjamins, Amsterdam-Philadelphia, 1996, pp. 115-149. 
tratar los conflictos fónicos y gramaticales en los malentendidos, las reglas constitutivas para describir las soluciones léxico-semánticas, y los mecanismos de reparación conversacional deben formularse por medio de instrucciones. Una paradoja interpretativa se cierne sobre las instrucciones de reparación: las instrucciones reparadoras se aplican rápidamente, y si se demoran en aplicarse es porque el conflicto pasó inadvertido. Por otra parte, las soluciones léxicas están ancladas en contextos muy inmediatos. Por fin, los malentendidos regulativos deberían ser tanto más improbables cuanto más sólidas sean las diferencias entre las variantes en conflicto.

Desde el punto de vista fónico, se documentan varias clases de operaciones diferentes: sustituciones, epéntesis, elisiones, fusiones, escisiones, cambios acentuales, cambios en la estructura silábica y cambios en la asignación del linde de palabra. A ellos hay que sumar algunos malentendidos fundamentados en problemas de comprensión transdialectal, en cambios de categoría y en la aparición o desaparición de material léxico. En las secciones en que ello es pertinente anoto primero los fenómenos vocálicos y luego los consonánticos.

\section{Sustituciones}

Una hipótesis preliminar haría esperar que sean más frecuentes los malentendidos cuantos más rasgos compartan dos fonemas. Veamos si hay indicios para pensar que esto es cierto ${ }^{11}$.

(5) Sólo un rasgo diferente

$$
\begin{aligned}
& \begin{array}{l}
a . \quad \mathrm{e} \quad \rightarrow \quad \mathrm{i}[23] \\
{[+ \text { alto }]}
\end{array}
\end{aligned}
$$

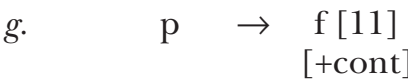

$$
\begin{aligned}
& \text { b. } \underset{\text { [+alto] }}{\mathrm{i}} \rightarrow \text { e [44] } \\
& \text { h. } \quad \mathrm{d} \rightarrow \mathrm{b} \text { [27] } \\
& \left(\begin{array}{l}
+ \text { cor } \\
+ \text { ant }
\end{array}\right)^{12} \quad(+ \text { lab }) \\
& \text { c. } \quad \mathrm{o} \quad \rightarrow \quad \mathrm{u}[13] \\
& i . \underset{(+\mathrm{lab})}{\mathrm{b}} \rightarrow \begin{array}{l}
\mathrm{d}[45] \\
\left.\begin{array}{l}
+\mathrm{cor} \\
+\mathrm{ant}
\end{array}\right)
\end{array}
\end{aligned}
$$

${ }^{11}$ Se acepta aquí el repertorio de rasgos propuesto en R. NúñEz CEDEÑo y A. MoRALES Front, Fonología generativa contemporánea de la lengua española, con la colab. de Pilar Prieto i Vives y José Ignacio Hualde, Georgetown University Press, Washington, 1999, p. 42. Los rasgos que están marcados con "+" en un fonema y con “-” en otro van entre corchetes, y los que quedan inespecificados en uno de los fonemas van entre paréntesis. Ahora bien, cambios en dos rasgos del segundo tipo se cuentan como uno solo si caen bajo el mismo nodo gestual. Por ejemplo, (+lab) $\rightarrow$ (+dorsal). 

d. $\underset{[+ \text { alto }]}{\mathrm{u}} \rightarrow \quad \mathrm{o}[40]$
j. $\quad \mathrm{k} \rightarrow \mathrm{t}[25]$
$(+$ dors $) \quad\left(\begin{array}{l}+ \text { cor } \\ + \text { ant }\end{array}\right)$
e. $\quad \underset{(+\mathrm{lab})}{\mathrm{b}} \rightarrow \underset{(+\mathrm{dors})}{\mathrm{g}[5]}$
k. $\quad \mathrm{k} \rightarrow \mathrm{x}[5]$
$[+$ cont $]$
f. $\quad \mathrm{p} \rightarrow \mathrm{b}[34]$
[+son]

\section{Como en:}

(6) L: Todo es más fácil si eres LOCAL.

P (entiende): Todo es más fácil en este Lugar.

P: Sí, pero todo es más caro.

L: Los locales tienen muchos descuentos.

P: Ah, los locales, decías los locales, creí que decías en este lugar [13].

(7) L: De todo lo que tengo lo sobrante me lo llevo a casa de mi mamá.

P (oye): De todo lo que tengo desodorante me lo llevo a casa de mi mamá [45].

(8) Dos rasgos diferentes

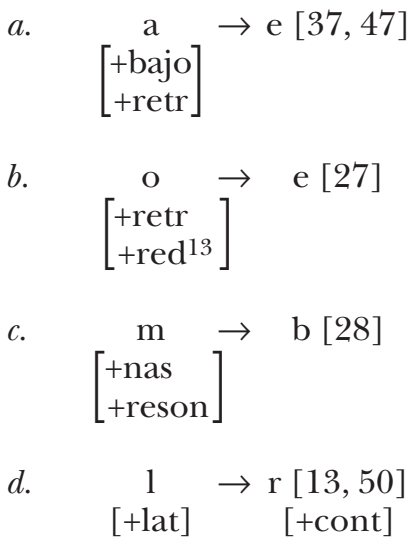

12 Cuento estos dos rasgos como uno solo en atención a que describen el PA de la $d$, de la misma manera que (+lab) describe el de la $b$. Este razonamiento se aplica también más adelante, entre otras cosas porque en los datos disponibles nunca se vio afectada una consonante -ant, aunque en ese caso de todos modos se hubiera anotado como [ant] y no como (ant), conforme al formalismo propuesto.

${ }^{13}$ El rasgo [red] podría no haberse considerado, y entonces esta regla formaría parte de la lista de un solo rasgo afectado; realmente, no altera la discusión que sigue. 
e. $\underset{\begin{array}{c}\text { [+son }] \\ \text { +dors })\end{array}}{\mathrm{g}} \rightarrow \quad \begin{gathered}\mathrm{t}[48] \\ \left.+\begin{array}{c}\text { +cor } \\ + \text { ant }\end{array}\right)\end{gathered}$

Un par de ejemplos:

(9) P: Yo creo que primero hacemos la TRAnscripción [tras].

Y: ¿Hacemos TREs qué? [47]

(10) Lu: ¿Cuándo vas a tejer tu Limón?

Pe (oye): ¿Cuándo vas a tejer tu RINCón? [50]

(11) Tres o más rasgos diferentes

a. $\left.\quad \begin{array}{c}\mathrm{n} \\ + \text { son } \\ + \text { nas } \\ + \text { reson }\end{array}\right] \rightarrow \begin{gathered}{[+ \text { cont }]^{14}} \\ \end{gathered}$

$\left.f . \quad \begin{array}{c}1 \\ + \text { son } \\ + \text { aprox } \\ + \text { lat } \\ + \text { reson }\end{array}\right]$
$\left(\begin{array}{c}+ \text { cor } \\ + \text { ant }\end{array}\right)$

$b$.

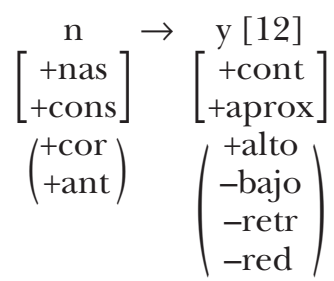

$g$.

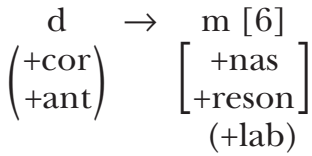

$c$.

$\mathrm{t} \quad \rightarrow \mathrm{n}[48]$

$\left[\begin{array}{c}+ \text { son } \\ + \text { nas } \\ + \text { reson }\end{array}\right]$

h. $\left.\quad \begin{array}{c}\mathrm{m} \\ + \text { son } \\ \text { +nas } \\ \text { +reson }\end{array}\right] \rightarrow \begin{gathered}\text { k [50] } \\ \text { (+dors })\end{gathered}$

$(+\mathrm{lab})$

d. nas $]$
n $\rightarrow \begin{gathered}1[2,27] \\ \left.\begin{array}{c}+ \text { aprox } \\ \text { +lat }\end{array}\right]\end{gathered}$

i. $\quad \mathrm{rr} \rightarrow \mathrm{g}$ [46]

$\left[\begin{array}{c}+ \text { cont } \\ + \text { aprox } \\ + \text { reson }\end{array}\right] \quad$ (+dors $)$

$\left(\begin{array}{c}+ \text { cor } \\ + \text { ant }\end{array}\right)$

14 No anoto (-distr) para /s/ puesto que los datos fueron recogidos en el contexto mexicano; es decir la /s/ no se opone a la $/ \theta /$ que es + distr. Esto en principio, pues hay algún ejemplo donde el conflicto se presenta precisamente por contacto dialectal. No es el caso del ejemplo al que se refiere esta regla, sin embargo. 
e.

$$
\underset{\left.\begin{array}{c}
\text { +aprox } \\
\text { +lat }
\end{array}\right]}{\rightarrow} \quad \begin{gathered}
\mathrm{n}[25] \\
{[+ \text { nas }]}
\end{gathered}
$$

Un solo ejemplo, pues esta situación ocurre nada más entre consonantes:

(12) Pe: Se está quedando gris la PELOTA.

L (oye): Se está quedando triste y PELONA

[una muñeca].

L: Sí, pero hay que lavarla.

Pe: ¿Cómo?

L: Ah, la pelota, entendí... la muñeca [48].

La sustitución de un segmento por otro afecta a casi la mitad de los ejemplos ${ }^{15}$. En principio, un solo rasgo se ve afectado en 11 procesos documentados en 11 ejemplos; 4 procesos son vocálicos y 7 consonánticos. De los 5 procesos y 7 ejemplos donde cambian 2 rasgos, 2 reglas afectan a vocales y 3 a consonantes; si se prescinde del rasgo de redondeamiento en las vocales, uno de esos procesos pasaría al listado de diferencias de un solo rasgo. Por fin, en el listado de tres o más rasgos diferentes aparecen 9 procesos documentados en 10 ejemplos, todos ellos consonánticos. Globalmente, hay 20 ejemplos de consonantes y 7 de vocales, que resultan ser mucho más estables. Todas las sustituciones vocálicas se dieron en posición átona. Por otra parte, por su naturaleza, las vocales del español sólo pueden diferir en muy pocos rasgos. Por tanto, las consonantes son las más interesantes para discutir si las sustituciones se dan más cuantos más rasgos se comparten. Pero resulta que tenemos 7 diferencias consonánticas de 1 rasgo, 3 de 2 y 9 de 3 o más. Si oponemos algo así como pocas diferencias a muchas diferencias, es decir 1 o 2 rasgos diferentes frente a 3 o más, tenemos 10 frente a 9 procesos. En términos puramente generales el simple número global de rasgos involucrados no afecta a las sustituciones en los malentendidos.

Sin embargo, hace mucho que la fonología ha postulado que existe una jerarquía de rasgos. Esquivando ahora la discusión de cuál es la jerarquía más adecuada ${ }^{16}$, y revisando sólo las consonantes, resulta que los nodos más afectados han sido los siguientes:

${ }^{15}$ La proporción es mayor si se considera que habría que restar de la cantidad total los malentendidos globales; ello supondría que más o menos en el $60 \%$ de los casos hay sustituciones.

16 Pero siguiendo de cerca la propuesta de G. N. Clements y E. Hume, "The internal organization of speech sounds", en The handbook of phonological theory, ed. J. A. Goldsmith, Basil Blackwell, Oxford, 1995, pp. 245-306, y el cap. 3 del libro citado de R. Núñez Cedeño y A. Morales-Front. 
(13) a. Consonantes obstruyentes

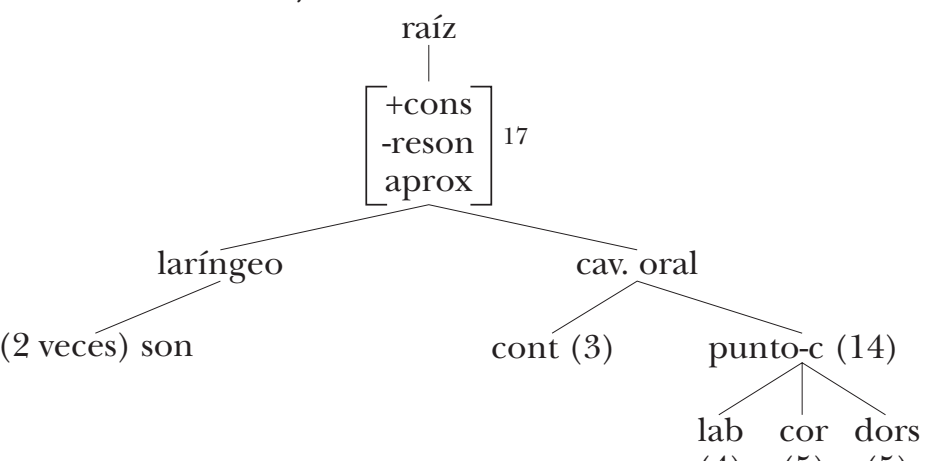

(4) (5) (5)

b. Nasales

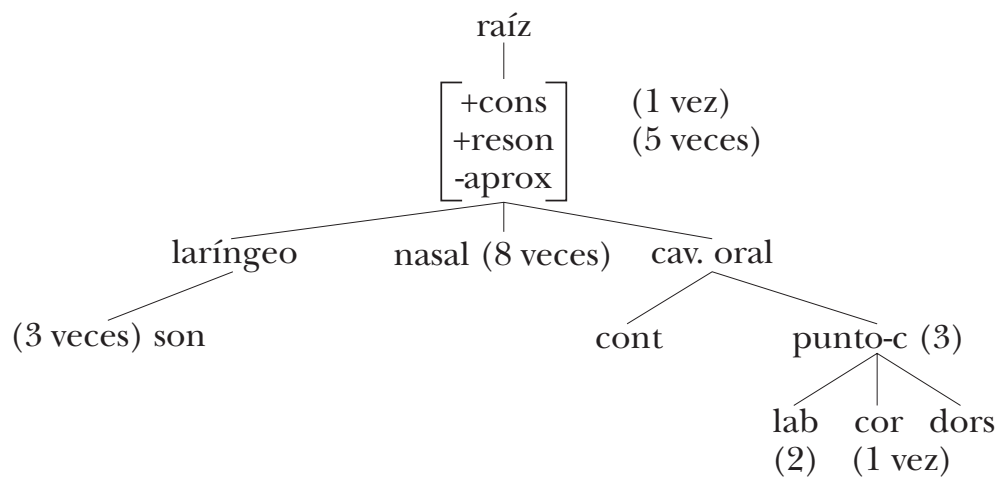

c. Líquidas

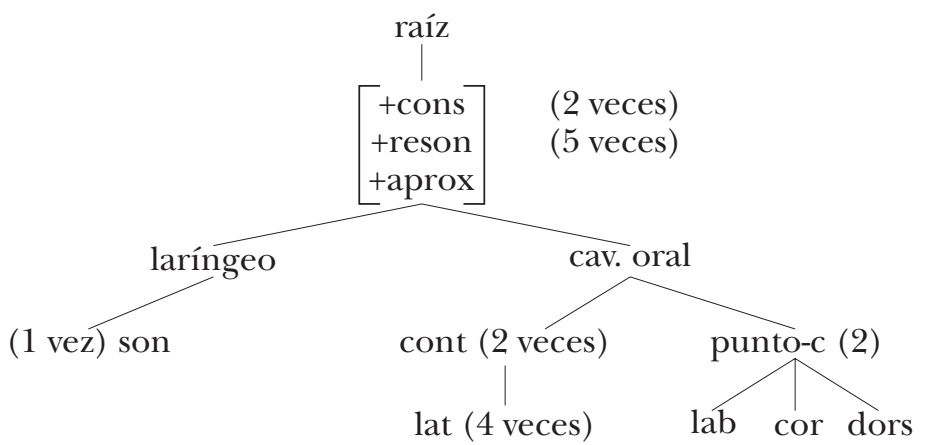

(2)

17 Clements y Hume colocan los rasgos resonante, aproximante y vocálico en la raíz (p. 292). Recuérdese que los sonidos obstruyentes son -resonantes, -aproximantes y -vocálicos, los nasales son +resonantes, -aproximantes y-vocálicos, los líquidos son +resonantes, +aproximantes y -vocálicos, y los sonidos vocálicos reciben + en los tres parámetros (Clements y Hume, p. 269; Núñez Cedeño y Morales-Front, p. 42). 
Los rasgos afectados más veces son los del punto-c o punto de constricción; en particular el rasgo [+cor] se hizo explícito en 8 de 19 ocasiones en que se hicieron patentes cambios en el punto-c ${ }^{18}$, así que parece ser el nodo más volátil; en general, lo es mucho más en las consonantes obstruyentes que en las nasales o en las líquidas. 28 procesos que afectan a rasgos se dieron en la cavidad oral; sumando las afecciones de la cavidad oral y la nasal, los articuladores supraglóticos concentraron 36 de los casos, en contraposición a los 6 del glótico. En cuanto a la raíz, los rasgos resonante ( 7 veces) y aproximante (5) fueron relativamente débiles, frente a la evidente solidez del rasgo consonántico. Las vocales concentraron las sustituciones en el punto vocálico. En cualquier caso, el talón de Aquiles de las obstruyentes parece ser la cavidad oral, en particular el punto-c; el de las nasales, la resonancia en la raíz y sobre todo la cavidad nasal; y en las líquidas, la raíz y el modo de articulación en la cavidad oral. Podrían proponerse por lo menos las siguientes generalizaciones:

(14) a. En la interpretación del oyente, las vocales tónicas no se sustituyen por otras .

b. Las vocales son más resistentes que las consonantes.

c. En la raíz, el rasgo consonántico es más sólido que los rasgos resonante y aproximante, en la interpretación del oyente.

d. El articulador glótico es más sólido que el articulador supraglótico.

e. En la cavidad oral, la continuidad, es decir el modo de articulación, MA, es más estable que el punto de articulación, PA, tanto al nivel del punto-c como del punto-v.

f. En la interpretación del punto-c, la región más inestable es la coronal anterior.

\section{Epéntesis}

Las epéntesis no son muy numerosas, pero responden a un patrón muy llamativo.

(15) Epéntesis vocálica

$$
\begin{aligned}
& a . \quad \varnothing \rightarrow \mathrm{o}[22,31,45] \\
& b . \quad \varnothing \rightarrow \mathrm{e}[21] \\
& c . \quad \varnothing \rightarrow \mathrm{a}[19]
\end{aligned}
$$

18 En términos de rasgos, aparece en 6 de los 7 procesos diferentes que se dieron en el punto-c. 
(16) Epéntesis consonántica

$$
\begin{aligned}
& a . \quad \varnothing \rightarrow \mathrm{s}[1,11,17,36] \\
& b . \quad \varnothing \rightarrow \mathrm{n}[50,44 \text { (2 veces) }] \\
& c . \quad \varnothing \rightarrow 1[46]
\end{aligned}
$$

El material epentético vocálico es hasta ahora [-alto]. Una segunda propiedad interesante es que las vocales que el oyente propone ocupan todas ellas posiciones [-tónicas], tal como se aprecia en ejemplos como los que siguen:

(17) $\mathrm{P}$ (dice a los niños): Estamos en Nunca JAMÁs.

L (entiende): Estamos en una caja más.

L: ¿Una caja? ¿UNA CAJA MÁs? ¿Qué quiere decir eso?

P: ¿Qué caja? ¿Qué caja dices?

L: La que dijiste, una caja más.

P (ríe): Ah no, no. Yo dije Nunca Jamás, la isla.

L: Yo entendí una caja más, como una caja grande pero en pequeño [19].

(18) Pe: A Alfonso LO PELARON.

Lu: ¿De dónde LO OPERARON?

L (ríe): No, no lo operaron. Lo pelaron [31].

Los datos cruciales ahora son la primera [a] de un[a] caja más, y la segunda [o] de lo [o]peraron. Un hecho interesante es que la reinterpretación del oyente desmonta reglas de contacto vocálico muy frecuentes, como en (18) ${ }^{19}$, donde se recupera el efecto que pudiera haber causado una hipotética regla de elisión de vocales ${ }^{20}$.

Más sorprendente todavía parece lo que ocurre con la epéntesis consonántica, a cargo exclusivamente de [s] y de [n] y en un caso de [1], como en

(19) P: XALAPA es bonita.

D (oye): LA PAZ [B. C.] es bonita.

D: ¿Cómo? ¿Que La Paz es bonita?

P (entiende): Que la paz es bonita.

P: No, Xalapa, es que como me puse solemne entendiste la paz.

D: No, la paz, no, La Paz, en Baja California [36].

${ }^{19}$ No es el único ejemplo de este tipo. Otro tanto ocurrió en un caso donde se pasó de sacabolas a se acabó qué [21], por ejemplo.

${ }^{20}$ Véase al respecto F. D'Introno, E. Del Teso y R. Weston, Fonética y fonología actual del español, Cátedra, Madrid, 1995, pp. 208 y ss., y pp. 223-224. 
A pesar de la escasez de datos, la generalización sería:

(20) El único material epentético autorizado por la interpretación del oyente son vocales [-altas] y consonantes [+cor, +ant].

\section{Elisiones}

Incluso un rápido vistazo a las elisiones revela un patrón de comportamiento bastante claro. Como hasta el momento no hay casos de elisión de vocales es posible formular la siguiente generalización ${ }^{21}$ :

(21) La interpretación del oyente no prescinde de las vocales.

Este hecho parece consistente con los escasos ejemplos de sustitución vocálica que es posible documentar, tal como se resumía en las generalizaciones $(14 a \mathrm{y} b)$.

No cualquier consonante parece susceptible de suprimirse en las contrapartes del interlocutor. Se pueden perder las siguientes, como se ve para (22b) en el ejemplo (23):

(22) Elisión consonántica
a. $\mathrm{m} \rightarrow \varnothing[6]$
b. $\mathrm{n} \rightarrow \varnothing[9,19,34]$
c. $1 \rightarrow \varnothing[1,37]$
d. $\mathrm{y} \rightarrow \varnothing[25]^{22}$

(23) L: La mala noticia es que voy a tener que hacer UN INFORME...

$\mathrm{P}$ (oye): La mala noticia es que voy a tener que hacer UNIFORME [están hablando de la escuela, cree que un uniforme del niño para que salga en la escolta].

L (prosigue): ...un informe por cada grupo [9].

Como estos sonidos forman un grupo natural, consonantes resonantes ${ }^{23}$, puede formularse la siguiente generalización:

(24) La interpretación del oyente sólo puede prescindir de las consonantes resonantes.

${ }^{21}$ No quiere decir que nunca se pierda material vocálico, pero cuando ocurre, es porque la vocal forma parte de una sílaba o de una palabra que se eliden, de manera que el fenómeno de elisión afecta en principio a ese tipo de unidades, no a la $\mathrm{V}$ en cuanto nodo.

${ }^{22}$ Supongo para ese caso que $y$ sí es [+cons].

23 También son sonoras, pero es rasgo redundante. 
Fusiones y escisiones

Una vez más se refuerza la idea de la solidez de las vocales, pues cabe formular esta generalización:

(25) La interpretación del oyente no funde ni escinde las vocales.

Con las consonantes, sin embargo, sí aparecen algunos, no muchos, casos de fusión y de escisión. Veámoslos.

(26) Fusión consonántica
a. $\mathrm{dr} \rightarrow 1[11]$
b. $\mathrm{gr} \rightarrow \mathrm{r}[41]$
c. $\lg \rightarrow \mathrm{x}[35]$

(27) Escisión consonántica
a. $\mathrm{t} \int \rightarrow \mathrm{ps}[23]$
b. $\mathrm{d} \rightarrow \mathrm{rg}[32]$

Los dos siguientes son el primero ejemplo de fusión, el segundo de escisión.

(28) L: PEDRI, ven aquí.

F: ¿Sí?

L: No, no, es a Pedrito. FELIS siempre cree que le hablo cuando llamo a Pedrito [11].

(29) P: Dos botellas de LECHE, por favor.

$\mathrm{T}$ (entiende): Dos botellas de PEPSI, por favor.

T: Pepsi no tengo, sólo Coca.

P: No, Pepsi, no, dos botellas, dos litros de leche. De leche, por favor [23].

Aunque hay pocos datos, pueden hacerse algunos comentarios. En todos los casos de fusión hay líquidas implicadas a la izquierda de la regla, y casi siempre a la derecha. De los dos casos de escisión, (27b) se parece a los de fusión en que aparece una líquida; en cuanto a $(27 a)$, no es difícil pensar que la solución $p s$ hereda la oclusión y la fricación de la t . Una vez más se constata el esfuerzo de correspondencia entre la izquierda y la derecha del malentendido. Una generalización posible, si bien apoyada en menos datos que en otras ocasiones, puede ser ésta:

(30) Los procesos de interpretación que conllevan fusiones y escisiones consonánticas suelen implicar a las consonantes líquidas (es decir a las +reson, +aprox). 


\section{Cambios acentuales}

Así como las vocales son bastante sólidas en el proceso de interpretación del oyente, otro tanto puede decirse de la asignación del acento. En muy pocos casos se altera. Hasta ahora, sólo en cuatro ejemplos se ve cambiado ${ }^{24}$.

(31) K: Te acuerdas de cómo tiene Marula así sus pelitos, ¿̇es cómo tengo yo también? Ya descubrí que es porque se baña para abajo. No me gusta. Mira cómo tengo, no me gusta, siento la frente MENOS LIBRE.

D: ¿ME LOS LIBRE?

K: Sí, menos libre.

D: ¡Ah! Yo entendí me los libre [2].

(32) L: Está FELIZ con sus juguetes.

$\mathrm{P}$ (oye): Está FELIS con sus juguetes.

P: ¿Qué...?

L: Que está feliz con sus juguetes, feliz.

P: Ah, feliz, entendí Felis, Felis está con sus juguetes [7].

(33) P: Un PAN MOLIDO, por favor.

A (dice algo desconcertada): No, palmolive no tengo. Sólo jabón Zote.

P: No, no. Pan molido.

A: Ah, ah [27].

En (31) y en (33) el acento primario se hace secundario -así que persiste en cierto modo-; sólo en (32) desaparece. Así que es factible proponer la siguiente generalización:

(34) La interpretación del oyente preserva la estructura acentual.

\section{Cambios en la estructura silábica}

Debe atenderse tanto al número de sílabas en cada contraparte del malentendido como a la estructura de cada sílaba particular. Como era de esperarse a la vista del sólido comportamiento de las vocales y de la estructura acentual, el número de sílabas casi nunca sufre alteraciones ${ }^{25}$. Sólo en contados casos hay mutaciones:

(35) P: La idea es un poco LOCA.

N: Sí, es LÓGICA.

P: No, no, no es lógica, digo que es loca [24].

${ }^{24}$ Los tres que aquí van, más el 44, que se copia más abajo con otro propósito. 
La estructura interna de la sílaba, en cambio, experimenta alteraciones más o menos en la tercera parte de los casos. Ahora bien, prácticamente siempre la parte afectada es la coda ${ }^{26}$, sea porque desaparece, $\mathrm{CVC} \rightarrow \mathrm{CV}-\mathrm{como}$ en $(36)-$, o porque aparece, $\mathrm{CV} \rightarrow \mathrm{CVC}$ -como en (37).

(36) L: La mala noticia es que voy a tener que hacer UN INFORME...

$\mathrm{P}$ (oye): La mala noticia es que voy a tener que hacer UNIFORME [están hablando de la escuela, cree que un uniforme del niño para que salga en la escolta].

L (prosigue): ...un informe por cada grupo [9].

(37) R: Son un problema los alumnos que están en el PATio.

L y otros maestros (oyen): Son un problema los alumnos que están en el PANTEÓN.

L y quizá otros: ¿En el panteón?

R: Están sentados en las jardineras [44].

En algún contado caso la parte afectada es la cabeza:

(38) L: ¿Qué me pondré porque me salió un BARRO?

LS (oye): ¿Qué me pondré porque me salió LumbaGo? [46]

Las consonantes implicadas en los malentendidos en las codas son siempre ${ }^{27}$ implosivas patrimoniales: $s, l, n$ (ya mencionadas antes, por cierto). Ahora bien, hay una diferencia entre ellas, ya anotada, la $l$ se elide (pero se añade en un ejemplo en la cabeza silábica), la $s$, se añade, y la $n$ puede elidirse o añadirse.

Propongamos algunas generalizaciones sobre la estructura silábica, sin importar lo provisionales que puedan resultar.

(39) $a$. El número de $\sigma$ de la grada silábica se mantiene en la interpretación del oyente.

b. El número de $\mathrm{C}$ y de $\mathrm{V}$ en la grada $\mathrm{CV}$ tiende a mantenerse en la interpretación del oyente, pero se producen algunas fluctuaciones en el número de $\mathrm{C}$ codas de origen patrimonial.

\section{Cambios en el linde de palabra}

Una proporción importante de los malentendidos —entre el 30 y el

${ }^{25}$ Sólo siete veces en medio centenar de ejemplos.

${ }^{26}$ Pocas veces hay alteraciones en la estructura de las cabezas silábicas: ya.ku.les $\rightarrow$ a.tu.nes [25], gru.po $\rightarrow$ ru.co [41]. Hay casos donde la cabeza silábica se altera, pero precisamente por afección obvia de la coda anterior: las alzas $\rightarrow$ las salsas [1].

${ }^{27}$ Hay por lo menos una excepción: leche $\rightarrow$ pepsi [23]. 
$40 \%$ de los casos, según se efectúe el recuento28 - están asociados a distorsiones en la división de palabras. En ocasiones \# aparece - como en (40)-, o se traslada - como en (41)-, pero en especial \# es propenso a perderse en la interpretación del oyente -como en (42).

(40) P: ¿Has visto el sacabolas? [de helado]

L: ¿Que SE ACABó qué?

P: No, digo el sacabolas de helado, así dije ahora [21].

(41) L: Me he enfermado mucho últimamente. Parece que tengo un CICLO PESADÍSIMO.

As (entienden): Tengo un cíclope SADísimo [el contexto es una clase de literatura precisamente sobre el Polifemo de Góngora, y otras versiones de fábulas mitológicas que L les está mostrando en libros de su propiedad: Carrillo, Villamediana, Jáuregui, Soto de Rojas, así que quizá lo interpretan como otro poema u otro libro].

As: ¿Cómo dices, un cíclope?

L: No, que tengo un ciclo pesadísimo [10].

(42) LF: LO SANO es hablar directamente.

P (entiende): LOZANO es... [la mala interpretación se rompe de inmediato; se hablaba de a quién habría que dirigirse acerca de un proyecto de investigación. P pensó que Lozano era la persona, y que no conocía a esa persona. Pero el equívoco se deshace de inmediato por lo que LF dice] [16].

A la vista de estos hechos, es factible proponer la siguiente generalización:

(43) La interpretación del oyente dispone de bastante libertad para asignar el linde de palabra (\#).

\section{Otros cambios}

Varios casos se explican primariamente por otras razones. Entre ellas, las principales son las diferencias dialectales de repertorio fo-

${ }^{28}$ En términos de lo realmente producido, algo más de $1 / 3$ de los casos implican modificaciones en la asignación de linde de palabra. Por otra parte, si consideramos que en dos palabras promedio del tipo $\sigma \sigma \sigma \# \sigma \sigma \sigma$ las posibilidades de partición léxica para el oyente son por lo menos $\sigma \# \sigma \sigma \sigma \sigma \sigma, \sigma \sigma \# \sigma \sigma \sigma \sigma, \sigma \sigma \sigma \# \sigma \sigma \sigma, \sigma \sigma \sigma \sigma \# \sigma \sigma$, $\sigma \sigma \sigma \sigma \sigma \# \sigma$, puede pensarse que hay cuando menos un $20 \%$ de acierto técnico casual en la asignación del linde, cuando el malentendido se ha localizado en otro nivel. Así que si se incluyera la corrección marcada por este sesgo, los casos asociados a asignaciones azarosas del linde de palabra supondrían el $40 \%$ o más del total de ejemplos. 
nológico, los cambios de categoría y las apariciones o desapariciones de material léxico.

Hay hasta el momento dos casos de cambio de categoría; en ambos casos la interpretación del oyente convierte en $\mathrm{N}$ lo que una vez era un $\mathrm{V}-(44)$ - y otra un Adj:

(44) L: ¿LIBRo el bote?

LS: Creí que te dejabas un LiBro [4].

Los cuatro ejemplos de malentendido por diferencias dialectales se deben a la presencia de $/ \theta /$ en el repertorio fonológico del oyente, pero no en el del hablante, de modo que se interpreta la /s/ producida como $/ \theta /$ :

(45) L: VOy a CERRAR [serrar] la puerta.

C (dice desconcertada): ¿Cómo?

$\mathrm{P}$ (dice aclarando): CERRAR, va a cerrar la puerta.

Por fin, en varias ocasiones los oyentes introdujeron o suprimieron palabras en sus comentarios, o el malentendido descansa obviamente en un problema léxico antes que fónico, como en (46), donde anda de por medio una mala interpretación metalingüística:

(46) Lu (dice, haciendo la tarea): ¿Cómo se dice DE en inglés?

L: ¿Cómo dices?

P: ¿¿De? De se dice DI. En inglés se dice di.

$\mathrm{Lu}$ (dice a L): Que cómo se dice de en inglés.

P: Di, se dice di.

L: Ah, es que ya ha dibujado al niño, boy, y de amarillo, yellow.

Lu: Yo quiero decir niño de amarillo.

P: Ah, de es of, pero...

\section{EL PROBLEMA FÓNICO Y LA SOLUCIÓN LÉXICA}

Aunque deba estudiarse con detenimiento cómo, quién y cuándo repara los malentendidos en la conversación, y no sea fácil establecer si la frontera entre malentendidos fónicos y léxicos es abrupta o gradual, pueden delimitarse las tres reglas sociolingüísticas más generales:

(47) a. Instrucción conversatoria: busca cualquier interpretación coherente con el contexto.

b. Regla constitutiva léxica: busca entender una palabra real.

c. Reglas regulativas fónicas: atiende a las generalizaciones expuestas.

(47a) es legítima si se considera que los casos documentados suelen descubrir su coherencia después de la reparación. Por otra parte, 
cabe pensar que los malentendidos que queden encubiertos para los participantes en una conversación son tanto más coherentes - pues por ello no se descubrieron ni se repararon.

(47b) está todavía más fuertemente apoyada en los datos recogidos. Salvo uno o dos casos, la mala interpretación buscó siempre entender una palabra que fuera real, que exista en el cuerpo léxico del español.

En cuanto a $(47 c)$, pueden hacerse algunas observaciones adicionales si se piensa el problema a partir del marco general que proporciona la teoría fonológica de la optimidad (TO ${ }^{29}$. Ciertos aspectos empíricos de los malos entendidos pueden poner a prueba algunos de los supuestos de la TO. Esta teoría propone la existencia de una serie de restricciones fonológicas universales que se aplican sobre el número infinito de candidatos posibles; en las lenguas particulares, las restricciones se encuentran jerarquizadas de una manera particular; esa jerarquía sirve para decidir cuál de los candidatos posibles es el óptimo. Uno de los atractivos de la TO es que permite tratar las excepciones. Lo interesante en los malentendidos es que no hay un solo candidato exitoso, sino que hay dos: el que A propuso y el que B entendió.

Una familia de restricciones dentro de la TO es la de las restricciones de correspondencia, que tienden a asegurar la conservación de la estructura o de algunos aspectos de ella. Dado que un malentendido es, finalmente, un problema de falta de correspondencia entre lo que A dijo en realidad y lo que B cree que dijo, es posible que la mayor parte de las generalizaciones propuestas hasta el momento puedan reducirse a una jerarquía de restricciones simple y comparable a la familia de restricciones de correspondencia de la TO. Repasemos las generalizaciones establecidas:

(14) a. Las vocales tónicas no son sustituidas por otras en la interpretación del oyente.

b. Las vocales son más resistentes que las consonantes.

c. En la raíz, el rasgo consonántico es más sólido que los rasgos resonante y aproximante, en la interpretación del oyente.

d. El articulador glótico es más sólido que el articulador supraglótico.

e. En la cavidad oral, la continuidad, es decir el modo de articulación, MA, es más estable que el punto de articulación, PA, tanto al nivel del punto-c como del punto-v.

f. En la interpretación del punto-c, la región más inestable es la coronal anterior.

29 Véase a este respecto el muy útil libro de D. ArChangeli y D. T. LANGENDoen (eds.), Optimality theory. An overview, Basil Blackwell, Oxford, 1997. 
(20) El único material epéntetico autorizado por la interpretación del oyente son vocales [-altas] y consonantes [+cor, +ant].

(21) La interpretación del oyente no prescinde de las vocales.

(24) La interpretación del oyente sólo puede prescindir de las consonantes resonantes.

(25) La interpretación del oyente no funde ni escinde las vocales.

(30) Los procesos de interpretación que conllevan fusiones y escisiones consonánticas suelen implicar a las consonantes líquidas (es decir a las +reson, +aprox).

(34) La interpretación del oyente preserva la estructura acentual.

(39) $a$. El número de $\sigma$ de la grada silábica se mantiene en la interpretación del oyente.

b. El número de $\mathrm{C}$ y de $\mathrm{V}$ en la grada $\mathrm{CV}$ tiende a mantenerse en la interpretación del oyente, pero se producen algunas fluctuaciones en el número de $\mathrm{C}$ codas de origen patrimonial.

(43) La interpretación del oyente dispone de bastante libertad para asignar el linde de palabra (\#).

En lo que respecta a las restricciones de correspondencia para la estructura de rasgos manejada, la jerarquía disponible es la siguiente:

(48) a. Corresp [-cons] > Corresp [+cons], o lo que es lo mismo, CorrespV $\gg$ CorrespC, a la vista de las generalizaciones $(14 b),(21)$ y $(25)$.

b. Corresp [+cons] > Corresp [+reson, +aprox], según (14c), (24) y (30).

c. CorrespSon (de sonoridad) $\gg$ Corresp cav. oral, según $(15 d)$.

d. Corresp MA $\gg$ Corresp PA, según (14e).

e. Corresp [-cor] $\gg$ Corresp [+cor], conforme a (14f) y a (20).

En cuanto a los rasgos suprasegmentales, lo que parece ocurrir es lo siguiente:

(49) a. Corresp [+tón] > Corresp [-ton], según (14a) y (34).

b. Corresp $[\sigma] \gg$ Corresp CV $\gg$ Corresp \#, a la vista de $(39 a)$, $(39 b)$ y $(43)$.

(48) y (49) son las generalizaciones regulativas que (47c) señala deben respetarse. 
Es poco probable que los malentendidos sean las causas o los efectos de la variación y el cambio lingüístico, aunque haya algunos casos en que esta idea sea defendible. En apariencia, muchos de los procesos de sustitución, epéntesis, elisión y otros documentados para los malentendidos no son exactamente como los procesos de variación y cambio que se suelen presentar en español, y cuando sí se trata de procesos de cambio naturales, no hay indicios obvios de que lo uno esté asociado con lo otro, el cambio con los malentendidos ${ }^{30}$. Los malentendidos se caracterizan también por tener una frecuencia de aparición relativamente baja. Los malentendidos son abruptos, discretos, los cambios son graduales.

Ahora bien, a la vista de la jerarquía de restricciones para los malentendidos propuesta en (48 y 49), resulta sugerente detenerse a pensar si al menos una parte de ella es aplicable a la variación y el cambio lingüístico manifiesto en el español contemporáneo (lo cual, debe insistirse, no implica que no haya excepciones): la solidez de las vocales o la inestabilidad del PA se encuentran entre las propiedades más generales de la variación fónica del español. ¿Por qué, entonces, podría darse esa connivencia, si no hay pruebas de causalidad en alguno de los sentidos? Probablemente la respuesta pueda empezar por ser ésta: los malentendidos y los cambios lingüísticos son manifestaciones diferentes de unos mismos principios de orden más abstracto, aquellos que rigen el umbral de maleabilidad de la teoría fonológica.

\section{Pedro Martín Butragueño El Colegio de México}

\footnotetext{
${ }^{30}$ Debe advertirse que Labov señala que "esos datos de malentendidos naturales no se emplean... para plantear que... son las causas del cambio" (op. cit., p. 430).
} 